Article

\title{
In Vitro Inhibitory Effects of Scutellarin on Six Human/Rat Cytochrome P450 Enzymes and P-glycoprotein
}

\author{
Yong-Long Han ${ }^{1}$, Dan $\mathrm{Li}^{1,2}$, Quan-Jun Yang ${ }^{1}$, Zhi-Yong Zhou ${ }^{1}$, Li-Ya Liu ${ }^{1,3}$, Bin Li ${ }^{1,3}$, \\ Jin Lu ${ }^{1}$ and Cheng Guo ${ }^{1, *}$
}

1 Department of Pharmacy, Shanghai Jiao Tong University Affiliated Sixth People's Hospital, No. 600 Yishan Road, Shanghai 200233, China

2 Department of Pharmacy, Wuhan University, Renmin Hospital, No.238 Jiefang Road, Wuchang disctrict, Wuhan 430060, China

3 Shanghai University of Traditional Chinese Medicine, No. 1200 Cailun Road, Shanghai 201203, China

* Author to whom correspondence should be addressed; E-Mail: guoc66@gmail.com; Tel.: +86-21-2405-8098; Fax: +86-21-2405-8445.

Received: 15 March 2014; in revised form: 25 April 2014 / Accepted: 25 April 2014 / Published: 5 May 2014

\begin{abstract}
Inhibition of cytochrome P450 (CYP) and P-glycoprotein (P-gp) are regarded as the most frequent and clinically important pharmacokinetic causes among the various possible factors for drug-drug interactions. Scutellarin is a flavonoid which is widely used for the treatment of cardiovascular diseases. In this study, the in vitro inhibitory effects of scutellarin on six major human CYPs (CYP1A2, CYP2C8, CYP2C9, CYP2C19, CYP2D6, and CYP3A4) and six rat CYPs (CYP1A2, CYP2C7, CYP2C11, CYP2C79, CYP2D4, and CYP3A2) activities were examined by using liquid chromatography-tandem mass spectrometry. Meanwhile, the inhibitory effects of scutellarin on P-gp activity were examined on a human metastatic malignant melanoma cell line WM-266-4 by calcein-AM fluorometry screening assay. Results demonstrated that scutellarin showed negligible inhibitory effects on the six major CYP isoenzymes in human/rat liver microsomes with almost all of the $\mathrm{IC}_{50}$ values exceeding $100 \mu \mathrm{M}$, whereas it showed values of $63.8 \mu \mathrm{M}$ for CYP2C19 in human liver microsomes, and 63.1 and 85.6 $\mu \mathrm{M}$ for CYP2C7 and CYP2C79 in rat liver microsomes, respectively. Scutellarin also showed weak inhibitory effect on P-gp. In conclusion, this study demonstrates that scutellarin is unlikely to cause any clinically significant herb-drug interactions in humans when co-administered with
\end{abstract}


substrates of the six CYPs (CYP1A2, CYP2C8, CYP2C9, CYP2C19, CYP2D6, and CYP3A4) and P-gp.

Keywords: scutellarin; cytochrome P450; P-glycoprotein; herb-drug interactions

\section{Introduction}

Pharmacokinetic drug-drug interactions (DDI) occur when a drug alters the absorption, distribution, metabolism, and excretion (ADME) of a co-administered drug, which may result in the increase or decrease of drug plasma concentration that can lead to serious adverse events or reduced drug efficacy. Cytochrome P450 (CYP), a superfamily of monooxygenases located primarily in hepatocytes, are the enzymes principally responsible for the metabolism of a lot of endogenous and exogenous compounds. It is generally accepted that $80 \%-90 \%$ of the clinical drugs are metabolized by CYP isoenzymes [1,2]. Of these, CYP3A4/5 accounts for approximately 30.2\%, CYP2D6 for 20\%, CYP2C 8 for 4.7\%, CYP2C9 for $12.8 \%$, CYP2C19 for $6.8 \%$ and CYP1A2 for $8.9 \%$ [2]. P-glycoprotein (P-gp), a $170 \mathrm{kDa}$ efflux glycoprotein encoded by the MDR-1 gene in humans belonging to the ABC superfamily, is localized in many tissues, including the apical surface of mature enterocytes, canalicular membranes of hepatocytes, and kidney and brain endothelial cells $[3,4]$. P-gp is responsible for the efflux of xenobiotics from cells and influences the pharmacokinetics of numerous drugs [5]. Alteration of CYP or P-gp activities involved in the absorption, distribution, metabolism, or excretion of a new compound by concomitant drugs maybe change drug exposure and affect drug response (safety or efficacy) [6].

Herbal medicines and their active ingredients are now widely used around the world and have become an important part of clinical medicine. In recent years, various studies have indicated that these phytochemicals have great potential to induce or inhibit the activities of CYP and/or P-gp, thus affecting the pharmacology and toxicology of the given drugs. A number of such herb-drug interactions (HDI) have been reported. For example, hyperoside is a potent selective CYP2D6 inhibitor in HLMs [7]. Baicalin can inhibit CYP2D and CYP3A activities in rat liver microsomes, and induce changes in the pharmacokinetics of midazolam and dextromethorphan in rats $[8,9]$. Rhinacanthin-C had an inhibitory effect on the expression and the function of P-gp, and the action was reversible [10]. Macelignan appeared to be effective to improve the cellular accumulation as well as oral exposure of paclitaxel, mainly via the inhibition of P-gp-mediated cellular efflux [11].

Scutellarin (Figure 1), a well-known flavone glycoside, is the primary active ingredient of an important Chinese traditional herbal medicine, Herba Erigerontis [Chinese name: Deng-zhan-xi-xin, Latin name: Erigeron breviscapus (Vant.) Hand.-Mazz.] belonging to the Compositae family that is mainly distributed in Yunnan Province of Southwest China and has been widely used as a folk remedy by the native people for the treatment of cardiovascular diseases and cerebrovascular diseases. Hence, many clinical preparations of Herba Erigerontis have been developed. Among these preparations, Herba Erigerontis injection (HEI), made from the aqueous extracts of Herba Erigerontis [12], is prominent for its remarkable curative effects for ischemic stroke, coronary heart disease and angina pectoris [13]. Our former study discovered that HEI can inhibit the activities of rat liver Cyp2d4 and Cyp3a2 in vivo [14]. Scutellarin is the capital effective component of HEI [12] and shows many 
significant beneficial pharmacological activities, such as antioxidant [15], anti-inflammatory [16-18], anti-diabetic complication [19], anti-hypertrophic [20], antiischemic [21,22], anti-obesity [23], anti-HIV-1 [24], anti-PRRSV [25], anti-tumor [26-31], hypercholesterolemia suppression [32], angiogenic [33], vasodilator [34], hepatoprotective [35] and neuroprotective effects [36-43]. Up to now, a lot of researches on the metabolism and pharmacokinetics of scutellarin in various models have been reported, but there is only one paper [44] that suggests that valsartan may inhibit the biliary excretion of scutellarin mediated by multidrug resistance-associated protein 2 and another paper [45] demonstrates that scutellarin, even at $100 \mathrm{mM}$, exerts weak inhibition towards the activity of UGT1A1, 1A6, 1A9 and 2B7 with 48.8\%, 20.2\%, 36.1\%, and 73.8\%, respectively. However, the studies of scutellarin concerning drug interactions based on CYPs and P-gp are rare. In addition, another result from acute and subacute toxicity studies suggests that scutellarin has a sufficient margin of safety for therapeutic use [46].

Figure 1. The structure of scutellarin.

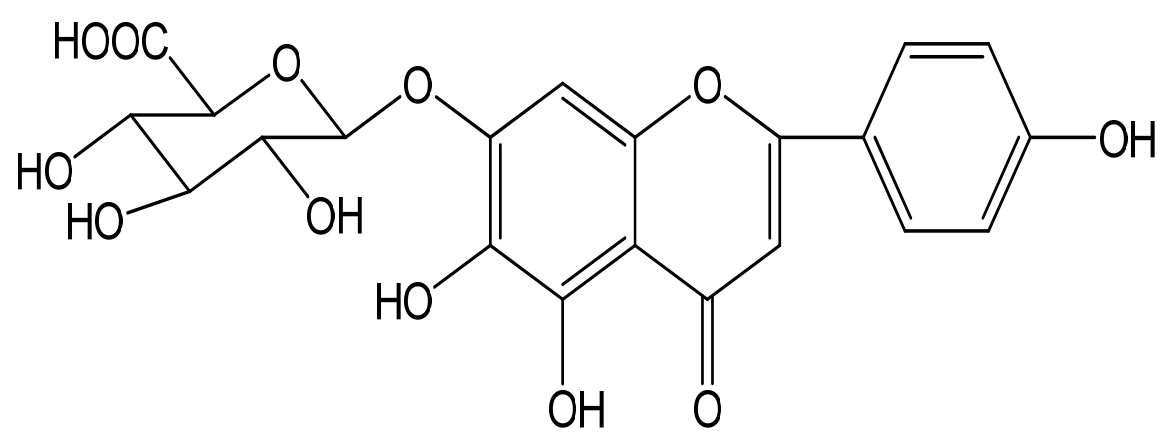

Consequently, we wanted to clarify whether scutellarin is responsible for the CYP inhibition activities of the HEI, even partly. At the same time, an increasing urgent demand of systematic investigation on HDI of scutellarin has been raised because of its wide applications. Therefore, the objective of this study was to systemically evaluate the in vitro inhibitory potential of scutellarin on the activities of six major cytochrome P450s (CYP1A2, CYP2C8, CYP2C9, CYP2C19, CYP2D6, and CYP3A4) and a transporter (P-gp) with the aim of avoidance of pharmacokinetic HDI, which was worthy of promoting safety and efficacy of scutellarin in the clinic.

\section{Results and Discussion}

The substrates and inhibitors of CYPs and P-gp used in this study were in line with the FDA's guideline and previous reports [47-50]. These experimental methods have been validated in our previous study $[51,52]$, and $\mathrm{IC}_{50}$ values of inhibitors were in good agreement with the published values according to the acceptable degree of accuracy [47-50].

Scutellarin was evaluated for the ability to inhibit the activities of the six CYPs (CYP1A2, CYP2C8, CYP2C9, CYP2C19, CYP2D6, and CYP3A4) and P-gp. The $\mathrm{IC}_{50}$ values for six CYPs in human/rat liver microsomes are presented in Table 1. P-gp inhibition is shown in Figure 2. 
Table 1. The $\mathrm{IC}_{50}$ values of scutellarin on the activities of six major CYP isoenzymes in HLM and RLM.

\begin{tabular}{ccccc}
\hline Isoenzyme & Substrate & Metabolites & $\begin{array}{c}\mathbf{I C}_{\mathbf{5 0}} \text { for } \mathbf{H L M} \\
(\boldsymbol{\mu M})\end{array}$ & $\begin{array}{c}\mathbf{I C}_{\mathbf{5 0}} \text { for } \mathbf{R L M} \\
(\boldsymbol{\mu M})\end{array}$ \\
\hline CYP1A2 & Phenacetin & Acetaminophen & $>100$ & $>100$ \\
CYP2C8 & Paclitaxel & 6a-Hydroxypaclitaxel & $>100$ & $63.1[95 \%$ CI: $39.3-101.3]$ \\
CYP2C9 & Diclofenac & 4-Hydroxydiclofenac & $>100$ & $>100$ \\
CYP2C19 & S-Mephenytoin & 4-Hydroxymephenytoin & 63.8 [95\% CI: $38.5-105.7]$ & $85.6[95 \%$ CI: $43.5-168.4]$ \\
CYP2D6 & Dextromethorphan & Dextrorphan & $>100$ & $>100$ \\
CYP3A4 & Midazolam & 1-Hydroxymidazolam & $>100$ & $>100$ \\
\hline
\end{tabular}

CI: Confidence Intervals.

Figure 2. Inhibition of P-gp by scutellarin (1000, 333.3, 111.1, 37.0, 12.3, and 4.1 $\mu \mathrm{M}$ ) using calcein AM assay. A response equal to or greater than $25 \%$ of verapamil at $100 \mu \mathrm{M}$ was considered to be positive at the tested concentration. Each data point represents the mean value $( \pm \mathrm{SD})$ of triplicate determinations. Experimental details are given in the section of P-glycoprotein assay.

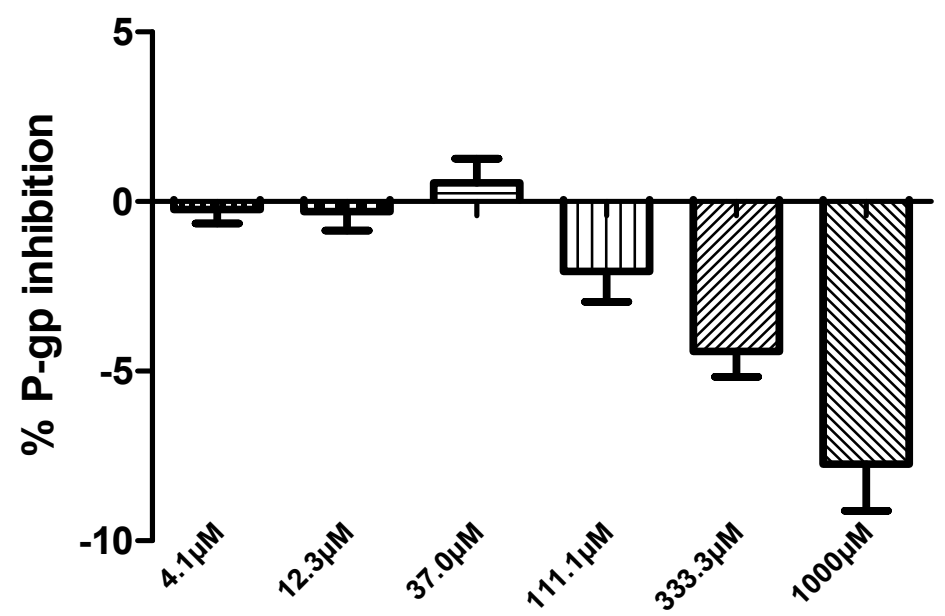

Scutellarin concentration

\subsection{Inhibition by Scutellarin on Six CYPs in HLM}

Using human liver microsomes and industry-accepted CYP substrates, scutellarin showed weak inhibitory effects on the six tested CYPs, as the $\mathrm{IC}_{50}$ values for CYP1A2, CYP2C8, CYP2C9, CYP2D6 and CYP3A4 were in excess of $100 \mu \mathrm{M}$, and only $63.8 \mu \mathrm{M}$ for CYP2C19 (Figure 3).

\subsection{Inhibition by Scutellarin on Six CYPs in RLM}

Similarly, scutellarin had a weak inhibitory effect on six tested CYPs using rat liver microsome, the $\mathrm{IC}_{50}$ values for CYP1A2, CYP2C11, CYP2D4 and CYP3A2 were in excess of $100 \mu \mathrm{M}$, but 63.1 and $85.6 \mu \mathrm{M}$ for CYP2C7 and CYP2C79 (Figure 4), respectively. 
Figure 3. Inhibition of human CYP1A2/2C8/2C9/2C19/2D6 and CYP3A4 by scutellarin $(100,30,10,3.0,1.0,0.3,0.1$, and $0.03 \mu \mathrm{M})$ using the enzyme/substrate cocktail method. The inhibitory effects of scutellarin on CYPs were shown in above figure respectively. Each data point represents the mean value $( \pm \mathrm{SD})$ of triplicate determinations. Experimental details are given in the section of CYP450 assay.
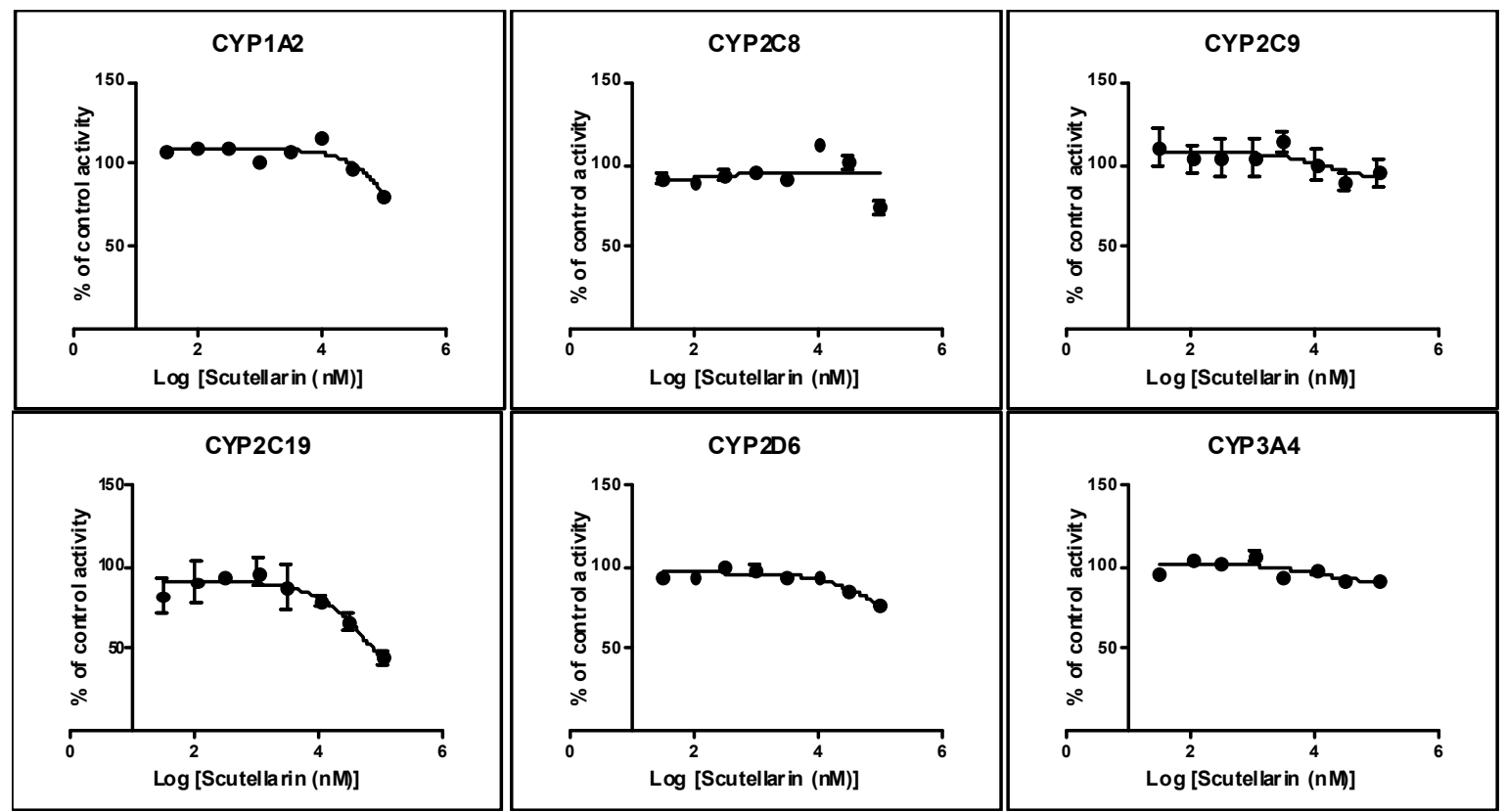

Figure 4. Inhibition of rat CYP1A2/2C7/2C11/2C79/2D4 and CYP3A2 by scutellarin $(100,30,10,3.0,1.0,0.3,0.1$, and $0.03 \mu \mathrm{M})$ using the enzyme/substrate cocktail method. The inhibitory effects of scutellarin on CYPs were shown in above figure respectively. Each data point represents the mean value $( \pm \mathrm{SD})$ of triplicate determinations. Experimental details are given in the section of CYP450 assay.
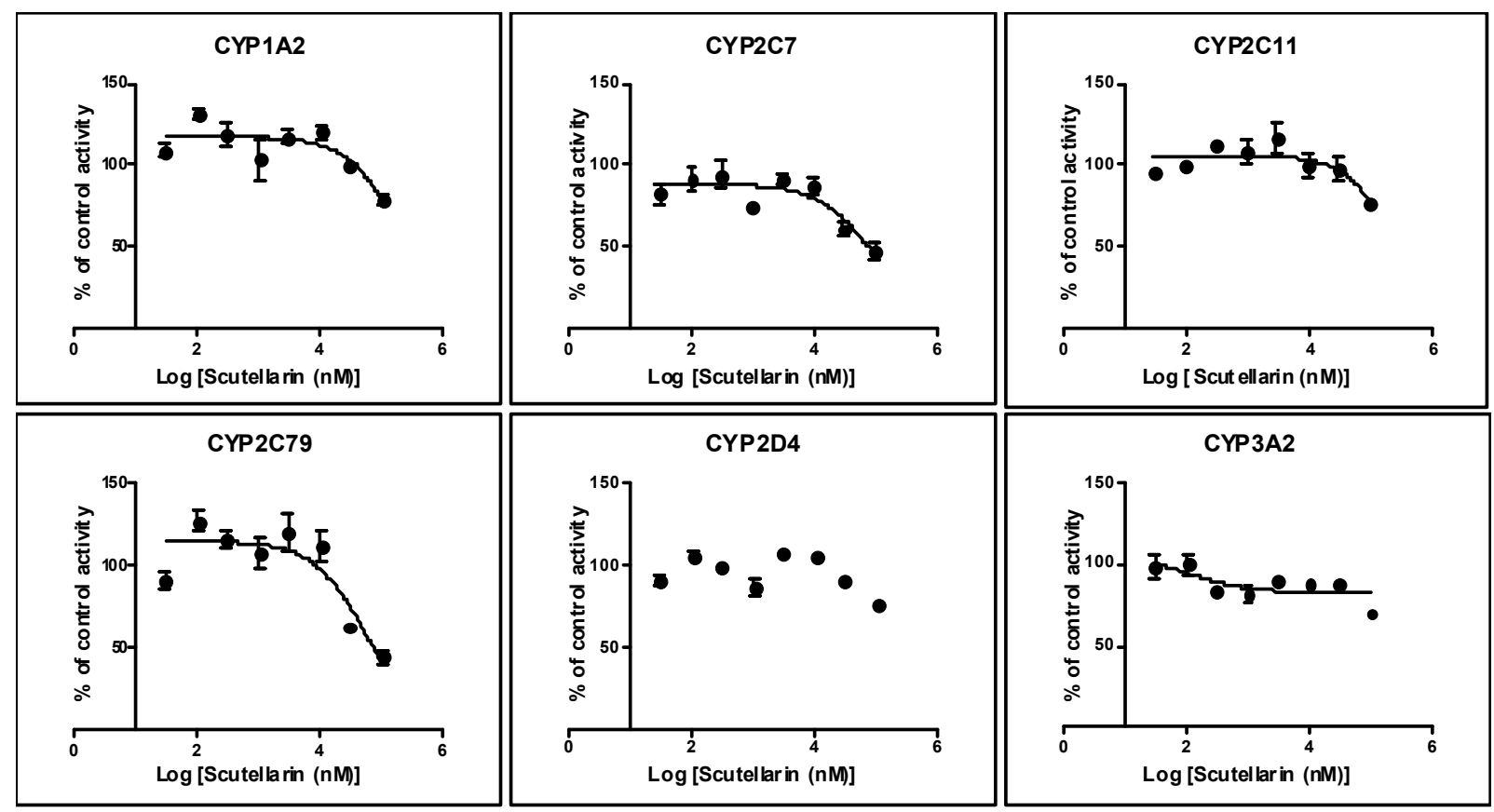


\subsection{Inhibition by Scutellarin on P-glycoprotein}

The effect of scutellarin on P-gp was examined by verapamil $(100 \mu \mathrm{M})$ as the positive control after results showed that scutellarin, even at highest concentration, does not show cytotoxicity on WM-266-4 cells. All of the six concentrations examined $(4.1 \sim 1,000 \mu \mathrm{M})$ for scutellarin produced less than $25 \%$ P-gp inhibition, which suggests that scutellarin cannot influence P-gp activity.

Our previous study discovered that HEI did not exert the inhibitory effects on rat liver CYP1A2, CYP2C11 and CYP2E1, but showed weak inhibitory activities on rat liver CYP2D4 and CYP3A2 in vivo [14]. Since scutellarin is the capital effective component of HEI [12] and it is necessary to investigate the in vitro inhibitory potential of scutellarin on CYP1A2, CYP2C7, CYP2C11, CYP2C79, CYP2D4, and CYP3A2 activities in RLM. These results showed that scutellarin (even at $100 \mu \mathrm{M}$ ) had no apparent inhibitory effects on rat CYP1A2, CYP2C11, CYP2D4 and CYP3A2 in vitro, but had weak inhibitory effects on rat CYP2C7 and CYP2C79 with $\mathrm{IC}_{50}$ values of 63.1 and $85.6 \mu \mathrm{M}$. Our present in vitro results were partly in accord with our former study, but our result on rat CYP1A2 was consistent with previous report that scutellarin was a poor inhibitor on rat CYP1A2 with an $\mathrm{IC}_{50}$ value of $108.20 \pm 0.657 \mu \mathrm{M}$ in in vitro experiments and exhibited a weak mixed-type inhibition against the activity of CYP1A2 with a $K_{i}$ value of $95.2 \mu \mathrm{M}$, Scutellarin at $30 \mathrm{mg} / \mathrm{kg}$ also weakly inhibited CYP1A2 activity in whole animal studies [53]. Because there were only the results of rat in all above studies, we studied in vitro inhibitory effects of scutellarin on human CYP1A2, CYP2C8, CYP2C9, CYP2D6 and CYP3A4. The in vitro results showed that scutellarin (even $100 \mu \mathrm{M}$ ) had no apparent inhibitory effects on human CYP1A2, CYP2C8, CYP2C9, CYP2D6 and CYP3A4 activities, but had weak inhibitory effects on human CYP2C19 activity with $\mathrm{IC}_{50}$ values of $63.8 \mu \mathrm{M}$. In our studies, there are no significant difference between the $\mathrm{IC}_{50}$ values of scutellarin on human/rat CYP2C8 and CYP2C19 which may be accepted for species differences. A compound that inhibits an isoform with $\mathrm{IC}_{50}$ value of less than $10 \mu \mathrm{M}$ is generally considered as a "potent" inhibitor to the isozyme, whereas the compound with $\mathrm{IC}_{50}$ value between 10 and $50 \mu \mathrm{M}$ is a "moderate" inhibitor [54,55]. Results from the in vitro studies can be used to predict in vivo interaction and guide the need for further in vivo study evaluation though it is not possible to directly extrapolate the in vitro results with what can occur in vivo. These viewpoints are widely accepted by researcher on CYP inhibition or drug interactions. So, we believed that scutellarin may have no significant inhibitory effects on CYP1A2, CYP2C8, CYP2C9, CYP2C19, CYP2D6, CYP3A4.

The WM-266-4 is an atypical cell line used in studies about drug interactions. Therefore, the method on P-gp inhibition-calceinAM assay was carefully validated in HD Biosciences Co., Ltd. and accordant with FDA's guideline. Furthermore, we found that scutellarin (even at the concentration of $1 \mathrm{mM}$ ) had no significant inhibitory effects on P-gp in WM-266-4 cells in this study.

\section{Experimental}

\subsection{Materials}

Scutellarin (LOT 110842-200605) was purchased from the National Institute for the Control of Pharmaceutical and Biological Products (Beijing, China). Phenacetin, diclofenac, midazolam, dextromethorphan, acetaminophen, furafylline, quinidine, 1-hydroxymidazolam, dextrorphan, sulfaphenazole, gemfibrozil, buspirone, calcein-AM, and verapamil were purchased from Sigma (St. Louis, 
MO, USA). Paclitaxel, S-mephenytoin, 4-hydroxymephenytoin, S-(+)-N-benzylnirvanol, montelukast, and ketoconazole were purchased from TRC (Toronto, ON, Canada). 6 $\alpha$-Hydroxypaclitaxel was purchased from Calbiochem (San Diego, CA, USA). 4-hydroxydiclofenac was purchased from BD Gentest Corporation (Woburn, MA, USA).

Pooled human liver microsomes (HLM, LOT 32556) were purchased from BD Gentest Corporation (simple donor information see Table 2.). Pooled rat liver microsomes were prepared from three male Sprague-Dawley rats in HD Biosciences Co. (Shanghai, China). The microsomal preparations contain a protein concentration of $10 \mathrm{mg} / \mathrm{mL}$ and are stored in a $-80{ }^{\circ} \mathrm{C}$ freezer before use. The human metastatic malignant melanoma cell line WM-266-4 was procured from ATCC (Manassas, VA, USA). Glucose-6-phosphate (G-6-P) and $\mathrm{NADP}^{+}$were purchased from Majorbio Co. (Shanghai, China). Glucose-6-phosphate dehydrogenase (G-6-PDH) was purchased from Calbiochem (Gibbstown, NJ, USA). The assay kit for protein determination with a biscinchoninic acid reagent was from Pierce (Rockford, IL, USA). Water was purified using a Milli-Q system (Millipore, Bedford, MA, USA) and used throughout the study. All inorganic salts were of analytical grade and were purchased from Sinopharm Chemical Reagent Co. (Shanghai, China). All organic solvents were of high performance liquid chromatography (HPLC) grade and were purchased from Sigma-Aldrich, USA.

Table 2. Simple information on fifty donors (Catalog No. 452156).

\begin{tabular}{ccccccccc}
\hline \multirow{2}{*}{ Unit } & \multicolumn{2}{c}{ Gender } & \multicolumn{4}{c}{ Age (From 21 to 77 Years) } & \multicolumn{2}{c}{ Race } \\
\cline { 2 - 9 } & Male & Female & $\leq \mathbf{3 9}$ & $\mathbf{4 0 \sim 4 9}$ & $\mathbf{5 0 ~ 5 9}$ & $\mathbf{2 6 0}$ & Caucasian & African Ameracian \\
\hline Number & 27 & 23 & 8 & 15 & 13 & 14 & 47 & 3 \\
\hline
\end{tabular}

\subsection{Instrument}

A Shimadzu LC-20A liquid chromatographic system equipped with a DGL-20A vacuum degasser, a dual pump, and a SIL-20A autosampler (Shimadzu, Tokyo, Japan) was used. Detection was performed using an API 4000 mass spectrometer equipped with a TurboIonSpray (ESI) Interface (Applied Biosystems, Concord, ON, Canada). The Analyst 1.5 software package (Applied Biosystems, Concord, ON, Canada) was used to control the LC-MS/MS system, and for data acquisition and processing. Fluorescence was measured by Flexstation II 96 Scanning Fluorometer (Molecular Devices, Sunnyvale, CA, USA).

\subsection{CYP450 Assay}

\subsubsection{Microsomal Incubations and Treatment Protocol}

Incubation mixtures were prepared in a total volume of $60 \mu \mathrm{L}$ with final component concentrations as follows: $0.1 \mathrm{M}$ potassium phosphate buffer ( $\mathrm{pH} 7.4$ ), $1.0 \mathrm{mM} \mathrm{NADP}{ }^{+}, 4.0 \mathrm{mM} \mathrm{MgCl}$, $10 \mathrm{mM}$ G-6-P, $1 \mathrm{U} / \mathrm{mL}$ G-6-PDH, $0.3 \mathrm{mg} / \mathrm{mL}$ human liver microsome or $0.5 \mathrm{mg} / \mathrm{mL}$ rat liver microsome, and Scutellarin (serial concentrations of $100,30,10,3.0,1.0,0.3,0.1$, and $0.03 \mu \mathrm{M}$ ) in triplicate. Positive controls (furafyline for CYP1A2, montelukast for CYP2C8, sulfaphenazole for CYP2C9, S-(+)-N-3-benzylnirvanol for CYP2C19, quinidine for CYP2D6, ketoconazole for CYP3A4) and specific substrates (phenacetin for CYP1A2, paclitaxel for CYP2C8, diclofenac for CYP2C9, S-mephenytoin for CYP2C19, dextromethorphan 
for CYP2D6, midazolam for CYP3A4) are consistent with our previous papers $[48,49]$. The substrates were used at concentrations approximately equal to their respective Michaelis-Menten constant $\left(\mathrm{K}_{\mathrm{m}}\right)$ values: $10 \mu \mathrm{M}$ phenacetin, $5 \mu \mathrm{M}$ paclitaxel, $5 \mu \mathrm{M}$ diclofenac, $200 \mu \mathrm{M}$ S-mephenytoin, $5 \mu \mathrm{M}$ dextromethorphan, and $2 \mu \mathrm{M}$ midazolam. $\mathrm{NADP}^{+}$was added after a 15 min preincubation of all other components at $37{ }^{\circ} \mathrm{C}$. After a given incubation time $(30 \mathrm{~min})$, the reactions were terminated by addition of $60 \mu \mathrm{L}$ ice-cold acetonitrile containing gemfibrozil and buspirone as internal standards. Incubation samples were stored at $-80{ }^{\circ} \mathrm{C}$ until used in liquid chromatography-tandem mass spectrometry (LC-MS/MS) analysis. The mixture was centrifuged at 3,500 rpm for $5 \mathrm{~min}$. The supernatant was mixed with equal volume of a 1:1 mixture of methanol and water and $20 \mu \mathrm{L}$ of the final mixture was used for LC-MS/ MS analysis.

\subsubsection{LC-MS/MS Analysis}

Chromatographic separations were performed using the Waters Nova-Pak ${ }^{\circledR} \mathrm{C}_{18}(150 \times 3.9 \mathrm{~mm})$ column. The column was maintained at ambient temperature $\left(25^{\circ} \mathrm{C}\right)$. A post-column diverter valve was used to direct the HPLC column eluate to a waste container for the first $3.2 \mathrm{~min}$ of the chromatographic run and then to the ionization source. The flow rate was maintained at $0.7 \mathrm{~mL} / \mathrm{min}$ and the mobile phase was used as follows: solvent A $[0.1 \%$ formic acid in mixture of acetonitrile/ methanol/formic acid (50/50/0.1)] and solvent $\mathrm{B}\left(5 \mathrm{mM} \mathrm{NH}_{4} \mathrm{Ac}\right)$.

The HPLC gradient program used was as follows: (1) mobile phase B was set to $5 \%$ at 0 min; (2) a linear gradient was run to $15 \% \mathrm{~B}$ in $2.0 \mathrm{~min}$; (3) a linear gradient was run to $80 \% \mathrm{~B}$ in $3.3 \mathrm{~min}$; (4) a linear gradient was run to $90 \% \mathrm{~B}$ in $3.6 \mathrm{~min}$; (5) a isocratic gradient was run to $90 \% \mathrm{~B}$ in $4.0 \mathrm{~min}$; (6) a linear gradient was run to $15 \% \mathrm{~B}$ in $7.0 \mathrm{~min}$; and (7) the solvent composition was returned to $5 \%$ $\mathrm{B}$ in $0.1 \mathrm{~min}$ for re-equilibration for $2 \mathrm{~min}$. The mass transitions of the metabolites of specific substrates using API4000 LC-MS/MS were consistent with our previous papers [48,49].

\subsection{P-glycoprotein Assay [49]}

One vial of WM-266-4 cells was thawed and cultured in a $15 \mathrm{~cm}$ culture dish overnight. Cells were split the next day and transferred onto a clear bottom 96-well plate with $5.0 \times 10^{4}$ cells per well. Scutellarin was serially diluted to concentrations of 2000, 666.7, 222.2, 74.1, 24.7, and 8.2 $\mu \mathrm{M}$ with a dilution factor of 3 , using culture media without serum. A volume of $50 \mu \mathrm{L}$ of each serial dilution of scutellarin was added to the 96-well plate in triplicate. The final concentrations of scutellarin in this reaction were $1000,333.4,111.1,37.1,12.4$, and $4.1 \mu \mathrm{M}$. Verapamil was used as a positive control at a concentration of $100 \mu \mathrm{M}$ and $1 \%$ DMSO was used as a negative control. After 30 min of incubation time, calcein-AM diluted with $50 \mu \mathrm{L}$ culture media without serum was added to a concentration of $1 \mu \mathrm{M}$ and the sample was incubated for an additional $30 \mathrm{~min}$. The plates were placed in the Flexstation II (Molecular Devices) and fluorescence was measured at excitation and emission wavelengths of 494 and $517 \mathrm{~nm}$, respectively. 


\subsection{Data Analysis}

The CYP inhibition potential were evaluated by measuring the formation of one or more metabolites of the test substrates. The peak area ratios of the metabolites and internal standard were acquired using the Analyst 1.5 software package (Applied Biosystems). The peak area ratios were plotted as a percentage of the relevant negative control for each reaction and the $50 \%$ inhibitory concentration $\left(\mathrm{IC}_{50}\right)$ values were calculated by nonlinear regression using Graphpad Prism 5.0 (San Diego, CA, USA).

The P-gp inhibitor verapamil was used as positive control at a concentration of $100 \mu \mathrm{M}$. The experiment was considered valid if an increase in fluorescence greater than 5-fold was observed. For P-gp inhibition, a compound with a response less than $25 \%$ of verapamil at $100 \mu \mathrm{M}$ is normally reported as "negative" at the tested concentration. The percent response value was used to determine and report the P-gp interaction at the tested concentration. A response equal to or greater than $25 \%$ of verapamil at $100 \mu \mathrm{M}$ was considered to be "positive" at the tested concentration.

\section{Conclusions}

In conclusion, the present study demonstrated that scutellarin showed no significant inhibitory effects on CYP1A2, CYP2C8, CYP2C9, CYP2C19, CYP2D6, CYP3A4 and P-gp, and it could not be the compound or a member of Herba Erigerontis injection which can inhibit CYP activities. Scutellarin is safe and unlikely to cause any clinically significant herb-drug interactions and thus cause the occurrence of adverse drug reactions in humans when co-adminstrated with subtrates of the six CYPs and P-gp.

\section{Acknowledgments}

This study was supported by the Traditional Chinese Medicine Scientific Research Fund of the Shanghai Municipal Health Bureau (No 2012L058A). We thank Xiao-yan Li and Gui-xuan Chai from HD Biosciences Co., Ltd. for their technical assistance.

\section{Author Contributions}

Guo and Han conceived the project and wrote the first draft. All authors contributed to data analysis and interpretations and to the writing of subsequent drafts. All authors have seen and approved the final version of the manuscript. All authors read and approved the final manuscript.

\section{Conflicts of Interest}

The authors declare no conflict of interest.

\section{References}

1. McGraw, J.; Waller, D. Cytochrome P450 variations in different ethnic populations. Expert. Opin. Drug Met. 2012, 8, 371-382. 
2. Zanger, U.M.; Schwab, M. Cytochrome P450 enzymes in drug metabolism: Regulation of gene expression, enzyme activities, and impact of genetic variation. Pharmacol. Ther. 2013, 138, 103-141.

3. Ambudkar, S.V.; Kimchi-Sarfaty, C.; Sauna, Z.E.; Gottesman, M.M. P-glycoprotein: From genomics to mechanism. Oncogene 2003, 22, 7468-7485.

4. Lin, J.H.; Yamazaki, M. Role of P-glycoprotein in pharmacokinetics: Clinical implications. Clin. Pharmacokinet. 2003, 42, 59-98.

5. Balayssac, D.; Authier, N.; Cayre, A.; Coudore, F. Does inhibition of P-glycoprotein lead to drug-drug interactions? Toxicol. Lett. 2005, 156, 319-329.

6. Zhang, L.; Reynolds, K.S.; Zhao, P.; Huang, S.M. Drug interactions evaluation: An integrated part of risk assessment of therapeutics. Toxicol. Appl. Pharmacol. 2010, 243, 134-145.

7. Song, M.; Hong, M.; Lee, M.; Jee, J.; Lee, Y.M.; Bae, J.S.; Jeong, T.C.; Lee, S. Selective inhibition of the cytochrome P450 isoform by hyperoside and its potent inhibition of CYP2D6. Food Chem. Toxicol. 2013, 59, 549-553.

8. Tian, X.; Cheng, Z.Y.; He, J.; Jia, L.J.; Qiao, H.L. Concentration-dependent inhibitory effects of baicalin on the metabolism of dextromethorphan, a dual probe of CYP2D and CYP3A, in rats. Chem. Biol. Interact. 2013, 203, 522-529.

9. Tian, X.; Cheng, Z.Y.; Jin, H.; Gao, J.; Qiao, H.L. Inhibitory effects of baicalin on the expression and activity of CYP3A induce the pharmacokinetic changes of midazolam in rats. Evid-Based Compl. Alt. 2013, 2013, doi:10.1155/2013/179643.

10. Wongwanakul, R.; Vardhanabhuti, N.; Siripong, P.; Jianmongkol, S. Effects of rhinacanthin-C on function and expression of drug efflux transporters in Caco-2 cells. Fitoterapia 2013, 89, 80-85.

11. Qiang, F.; Lee, B.J.; Ha, I.; Kang, K.W.; Woo, E.R.; Han, H.K. Effect of maceligan on the systemic exposure of paclitaxel: In vitro and in vivo evaluation. Eur. J. Pharm. Sci. 2010, 41, 226-231.

12. National Commission of Chinese Pharmacopoeia. Pharmacopoeia of the People's Republic of China; Chemical Industry Press: Beijing, China, 2010; pp. 707-708.

13. Liao, S.G.; Zhang, L.J.; Li, C.B.; Lan, Y.Y.; Wang, A.M.; Huang, Y.; Zhen, L.; Fu, X.Z.; Zhou, W.; Qi, X.L.; et al. Rapid screening and identification of caffeic acid and its esters in Erigeron breviscapus by ultra-performance liquid chromatography/tandem mass spectrometry. Rapid Commun. Mass Sp. 2010, 24, 2533-2541.

14. Han, Y.L.; Li, D.; Ren, B.; Jing, G.P.; Meng, X.L.; Zhou, Z.Y.; Yu, Q.; Li, Y.; Wan, L.L.; Guo, C. Evaluation of impact of Herba Erigerontis injection, a Chinese herbal prescription, on rat hepatic cytochrome P450 enzymes by cocktail probe drugs. J. Ethnopharmacol. 2012, 139, 104-109.

15. Hong, H.; Liu, G. Protection against hydrogen peroxide-induced cytotoxicity in PC12 cells by scutellarin. Life Sci. 2004, 74, 2959-2973.

16. Chen, H.; Zhang, X.; Ou, J.; Jiang, J.; Wu, D. Research on scutellarin parenteral solution's protective effects in rats with severe acute pancreatitis and multiple organ injuries. Inflammation 2012, 35, 1005-1014. 
17. Luo, P.; Zhang, Z.; Yi, T.; Zhang, H.; Liu, X.; Mo, Z. Anti-inflammatory activity of the extracts and fractions from Erigeron multiradiatus through bioassay-guided procedures. J. Ethnopharmacol. 2008, 119, 232-237.

18. Luo, P.; Tan, Z.H.; Zhang, Z.F.; Zhang, H.; Liu, X.F.; Mo, Z.J. Scutellarin isolated from Erigeron multiradiatus inhibits high glucose-mediated vascular inflammation. Yakuga. Zasshi 2008, 128, 1293-1299.

19. Su, Y.; Liu, W.; Ma, L.; Liu, X.; Liu, Z.; Zhu, B. Scutellarin inhibits translocation of protein kinase $\mathrm{C}$ in diabetic thoracic aorta of the rat. Clin. Exp. Pharmacol. Physiol. 2012, 39, 136-140.

20. Pan, Z.W.; Zhang, Y.; Mei, D.H.; Zhang, R.; Wang, J.H.; Zhang, X.Y.; Xu, C.Q.; Lu, Y.J.; Yang, B.F. Scutellarin exerts its anti-hypertrophic effects via suppressing the $\mathrm{Ca}^{+}$-mediated calcineurin and CaMKII signaling pathways. Naunyn Schmiedebergs Arch. Pharmacol. 2010, $381,137-145$.

21. Lin, L.L.; Liu, A.J.; Liu, J.G.; Yu, X.H.; Qin, L.P.; Su, D.F. Protective effects of scutellarin and breviscapine on brain and heart ischemia in rats. J. Cardiovasc. Pharmacol. 2007, 50, 327-332.

22. Zhang, G.; Qiu, S.; Wei, H. Scutellarin blocks sodium current in freshly isolated mouse hippocampal CA1 neurons. Neurochem. Res. 2011, 36, 947-54.

23. Lu, K.; Han, M.; Ting, H.L.; Liu, Z.; Zhang, D. Scutellarin from Scutellaria baicalensis suppresses adipogenesis by upregulating PPAR $\alpha$ in 3T3-L1 cells. J. Nat. Prod. 2013, 76, 672-678.

24. Zhang, G.H.; Wang, Q.; Chen, J.J.; Zhang, X.M.; Tam, S.C.; Zheng, Y.T. The anti-HIV-1 effect of scutellarin. Biochem. Biophys. Res. Commun. 2005, 334, 812-816.

25. Bai, X.; Sun, N.; Zhao, X.; Niu, L.; Song, M.; Sun, Y.; Jiang, J.; Guo, J.; Bai, Y.; He, J.; et al. In vitro Screening for compounds derived from traditional Chinese medicines (TCMs) with antiviral activities against porcine reproductive and respiratory syndrome virus. J. Microbiol. Biotechnol. 2013, 23, 1076-1083.

26. Feng, Y.; Zhang, S.; Tu, J.; Cao, Z.; Pan, Y.; Shang, B.; Liu, R.; Bao, M.; Guo, P.; Zhou, Q. Novel function of scutellarin in inhibiting cell proliferation and inducing cell apoptosis of human Burkitt lymphoma Namalwa cells. Leuk. Lymphoma 2012, 53, 2456-2464.

27. Li, H.; Huang, D.; Gao, Z.; Chen, Y.; Zhang, L.; Zheng, J. Scutellarin inhibits the growth and invasion of human tongue squamous carcinoma through the inhibition of matrix metalloproteinase-2 and -9 and $\alpha v \beta 6$ integrin. Int. J. Oncol. 2013, 42, 1674-1681.

28. Li, H.; Fan, H.; Wang, Z.; Zheng, J.; Cao, W. Potentiation of scutellarin on human tongue carcinoma xenograft by low-intensity ultrasound. PLoS One 2013, 8, e59473.

29. Xu, H.; Zhang, S. Scutellarin-induced apoptosis in HepG2 hepatocellular carcinoma cells via a STAT3 pathway. Phytother. Res. 2013, 27, 1524-1528.

30. Yang, B.; Zhao, Y.L.; Yang, X.; Liao, X.L.; Yang, J.; Zhang, J.H.; Gao, C.Z. Scutellarin-cyclodextrin conjugates: Synthesis, characterization and anticancer activity. Carbohydr. Polym. 2013, 92, 1308-1314.

31. Wu, Y.X.; Sato, E.; Kimura, W.; Miura, N. Baicalin and scutellarin are proteasome inhibitors that specifically target chymotrypsin-like catalytic activity. Phytother. Res. 2013, 27, 1362-1367.

32. Li, Q.; Wu, J.H.; Guo, D.J.; Cheng, H.L.; Chen, S.L.; Chan, S.W. Suppression of diet-induced hypercholesterolemia by scutellarin in rats. Planta Med. 2009, 75, 1203-1208. 
33. Gao, Z.X.; Huang, D.Y.; Li, H.X.; Zhang, L.N.; Lv, Y.H.; Cui, H.D.; Zheng, J.H. Scutellarin promotes in vitro angiogenesis in human umbilical vein endothelial cells. Biochem. Biophys. Res. Commun. 2010, 400, 151-156.

34. Pan, Z.; Feng, T.; Shan, L.; Cai, B.; Chu, W.; Niu, H.; Lu, Y.; Yang, B. Scutellarin-induced endothelium-independent relaxation in rat aorta. Phytother. Res. 2008, 22, 1428-1433.

35. Tan, Z.H.; Yu, L.H.; Wei, H.L.; Liu, G.T. The protective action of scutellarin against immunological liver injury induced by concanavalin A and its effect on pro-inflammatory cytokines in mice. J. Pharm. Pharmacol. 2007, 59, 115-121.

36. Chai, L.; Guo, H.; Li, H.; Wang, S.; Wang, Y.L.; Shi, F.; Hu, L.M.; Liu, Y.; Adah, D. Scutellarin and caffeic acid ester fraction, active components of Dengzhanxixin injection, upregulate neurotrophins synthesis and release in hypoxia/reoxygenation rat astrocytes. J. Ethnopharmacol. 2013, 150, 100-107.

37. Guo, H.; Hu, L.M.; Wang, S.X.; Wang, Y.L.; Shi, F.; Li, H.; Liu, Y.; Kang, L.Y.; Gao, X.M. Neuroprotective effects of scutellarin against hypoxic-ischemic-induced cerebral injury via augmentation of antioxidant defense capacity. Chin. J. Physiol. 2011, 54, 399-405.

38. Guo, L.L.; Guan, Z.Z.; Wang, Y.L. Scutellarin protects against A $\beta$-induced learning and memory deficits in rats: Involvement of nicotinic acetylcholine receptors and cholinesterase. Acta Pharm. Sin. 2011, 32, 1446-1453.

39. Guo, L.L.; Guan, Z.Z.; Huang, Y.; Wang, Y.L.; Shi, J.S. The neurotoxicity of $\beta$-amyloid peptide toward rat brain is associated with enhanced oxidative stress, inflammation and apoptosis, all of which can be attenuated by scutellarin. Exp. Toxicol. Pathol. 2013, 65, 579-584.

40. Li, J.H.; Lu, J.; Zhang, H. Functional recovery after scutellarin treatment in transient CEREBRAL ischemic rats: A pilot study with 18 F-fluorodeoxyglucose micropet. Evid. Based Complement. Alternat. Med. 2013, 2013, doi:10.1155/2013/507091.

41. Xu, W.; Zha, R.P.; Wang, W.Y.; Wang, Y.P. Effects of scutellarin on PKCgamma in PC12 cell injury induced by oxygen and glucose deprivation. Acta Pharm. Sin. 2007, 28, 1573-1579.

42. Zhu, J.; Choi, R.; Li, J.; Xie, H.; Bi, C.; Cheung, A.; Dong, T.; Jiang, Z.; Chen, J.; Tsim, K. Estrogenic and neuroprotective properties of scutellarin from Erigeron breviscapus: A drug against postmenopausal symptoms and Alzheimer's disease. Planta Med. 2009, 75, 1489-1493.

43. Wang, S.; Wang, H.; Guo, H.; Kang, L.; Gao, X.; Hu, L. Neuroprotection of scutellarin is mediated by inhibition of microglial inflammatory activation. Neuroscience 2011, 185, 150-160.

44. Cui, M.Y.; Tian, C.C.; Ju, A.X.; Zhang, C.T.; Li, Q.H. Pharmacokinetic interaction between scutellarin and valsartan in rats. Acta Pharm. Sin. 2013, 48, 541-546.

45. Ma, G.Y.; Cao, Y.F.; Hu, C.M.; Fang, Z.Z.; Sun, X.Y.; Hong, M.; Zhu, Z.T. Comparison of Inhibition Capability of Scutellarein and Scutellarin Towards Important Liver UDPGlucuronosyltransferase (UGT) Isoforms. Phytother. Res. 2014, 28, 382-386.

46. Li, X.; Wang, L.; Li, Y.; Bai, L.; Xue, M. Acute and subacute toxicological evaluation of scutellarin in rodents. Regul. Toxicol. Pharm. 2011, 60, 106-111.

47. Guidance for Industry: Drug interaction studies-study design, data analysis, and implications for dosing and labelling recommendations. Available online: http://www.fda.gov/downloads/Drugs/ GuidanceComplianceRegulatoryInformation/Guidances/UCM292362.pdf (accessed on 4 December 2013). 
48. Pelkonen, O.; Turpeinen, M.; Hakkola, J.; Honkakoski, P.; Hukkanen, J.; Raunio, H. Inhibition and induction of human cytochrome P450 enzymes: Current status. Arch. Toxicol. 2008, 82, $667-715$.

49. Walsky, R.L.; Obach, R.S. Verification of the selectivity of $(+) N$-3-benzylnirvanol as a CYP2C19 inhibitor. Drug Metab. Dispos. 2003, 31, 343.

50. Yuan, R.; Madani, S.; Wei, X.X.; Reynolds, K.; Huang, S.M. Evaluation of cytochrome P450 probe substrates commonly used by the pharmaceutical industry to study in vitro drug interactions. Drug Metab. Dispos. 2002, 30, 1311-1319.

51. Han, Y.L.; Yu, H.L.; Meng, X.L.; Li, D.; Zhou, Z.Y.; Yu, Q.; Wang, F.J.; Zhang, X.Y.; Guo, C. In vitro inhibition of Huanglian [Rhizoma coptidis (L.)] and its six active alkaloids on six cytochrome P450 isoforms in human liver microsomes. Phytother. Res. 2011, 25, 1660-1665.

52. Han, Y.L.; Yu, H.L.; Meng, X.L.; Li, D.; Zhou, Z.Y.; Yu, Q.; Wang, F.J.; Zhang, X.Y.; Guo, C. Inhibitory effects of limonin on six human cytochrome P450 enzymes and P-glycoprotein in vitro. Toxicol. In Vitro 2011, 25, 1828-1833.

53. Jian, T.Y.; He, J.C.; He, G.H.; Feng, E.F.; Li, H.L.; Bai, M.; Xu, G.L. Scutellarin inhibits cytochrome P450 isoenzyme 1A2 (CYP1A2) in rats. Phytother. Res. 2013, 26, 1226-1230.

54. Dierks, E.A.; Stams, K.R.; Lim, H.K.; Cornelius, G.; Zhang, H.; Ball, S.E. A method for the simultaneous evaluation of the activities of seven major human drug-metabolizing cytochrome P450s using an in vitro cocktail of probe substrates and fast gradient liquid chromatography tandem mass spectrometry. Drug Metab. Dispos. 2001, 29, 23-29.

55. Herzke, D.; Thiel, R.; Rotard, W.D.; Neubert, D. Kinetics and organotropy of some polyfluorinated dibenzo-p-dioxins and dibenzofurans (PFDD/PFDF) in rats. Life Sci. 2002, 71, 1475-1486.

Sample Availability: Samples of the compounds and materials are available from the authors.

(C) 2014 by the authors; licensee MDPI, Basel, Switzerland. This article is an open access article distributed under the terms and conditions of the Creative Commons Attribution license (http://creativecommons.org/licenses/by/3.0/). 